\title{
Width and mean curvature flow
}

\author{
TOBIAS H COLDING \\ WiLliAM P MINICOZZI II
}

\begin{abstract}
Given a Riemannian metric on a homotopy $n$-sphere, sweep it out by a continuous one-parameter family of closed curves starting and ending at point curves. Pull the sweepout tight by, in a continuous way, pulling each curve as tight as possible yet preserving the sweepout. We show: Each curve in the tightened sweepout whose length is close to the length of the longest curve in the sweepout must itself be close to a closed geodesic. In particular, there are curves in the sweepout that are close to closed geodesics.
\end{abstract}

As an application, we bound from above, by a negative constant, the rate of change of the width for a one-parameter family of convex hypersurfaces that flows by mean curvature. The width is loosely speaking up to a constant the square of the length of the shortest closed curve needed to "pull over" $M$. This estimate is sharp and leads to a sharp estimate for the extinction time; cf our papers $[7 ; 8]$ where a similar bound for the rate of change for the two dimensional width is shown for homotopy 3-spheres evolving by the Ricci flow (see also Perelman [13]).

$53 \mathrm{C} 44,58 \mathrm{E} 10 ; 53 \mathrm{C} 22$

\section{Introduction}

Given a Riemannian metric on the 2-sphere, sweep the 2 -sphere out by a continuous one-parameter family of closed curves starting and ending at point curves. Pull the sweepout tight by, in a continuous way, pulling each curve as tight as possible yet preserving the sweepout. We show the following useful property; see Theorem 1.5 below and cf our papers [7; 8], Proposition 3.1 of Colding and De Lellis [5], Proposition 3.1 of Pitts [14] and 12.5 of Almgren [1]:

Each curve in the tightened sweepout whose length is close to the length of the longest curve in the sweepout must itself be close to a closed geodesic. In particular, there are curves in the sweepout that are close to closed geodesics. 
Finding closed geodesics on the 2 -sphere by using sweepouts goes back to Birkhoff in 1917; see Birkhoff [2;3] and Section 2 in Croke [9] about Birkhoff's ideas. The argument works equally well on any closed manifold, but only produces nontrivial closed geodesics when the width, which is defined in (1.1) below, is positive. For instance, when $M$ is topologically a 2 -sphere, the width is loosely speaking up to a constant the square of the length of the shortest closed curve needed to "pull over" $M$. Thus Birkhoff's argument gives that $2 \pi$ times the width is realized as the length squared of a closed geodesic.

The above useful property is virtually always implicit in any sweepout construction of critical points for variational problems yet it is not always recorded since most authors are only interested in the existence of one critical point.

Similar results holds for sweepouts of manifolds by 2-spheres instead of circles; cf our paper [8]. The ideas are essentially the same in the two cases, though the techniques in the curve case are purely ad hoc whereas in the 2 -sphere case additional techniques, developed in the 1980s, have to be used to deal with energy concentration (ie, "bubbling"); cf Jost [12].

As an application of the main result, we bound from above, by a negative constant, the rate of change of the width for a one-parameter family of convex hypersurfaces that flows by mean curvature. This estimate is sharp and leads to a sharp estimate for the extinction time; cf $[7 ; 8]$ where a similar bound for the rate of change for the two-dimensional width is shown for homotopy 3-spheres evolving by the Ricci flow (see also Perelman [13]).

\section{Existence of good sweepouts by curves}

Let $M$ be a closed Riemannian manifold and $W^{1,2}$ the space of $W^{1,2}$ maps from $\mathbf{S}^{1}$ to $M$. We will use the distance and topology on $W^{1,2}$ given by the $W^{1,2}$ (Sobolev) norm. The simplest way to define the $W^{1,2}$ norm is to isometrically embed the compact manifold $M$ into some Euclidean space $\mathbf{R}^{N} .^{1}$ It will be convenient to scale $\mathbf{R}^{N}$, and thus $M$, by a constant so that it satisfies the following:

(M1) $\sup _{M}|A| \leq 1 / 16$, where $|A|^{2}$ is the norm squared of the second fundamental form of $M$, ie, the sum of the squares of the principal curvatures (see, eg, (1.24) on page 4 of [6]);

\footnotetext{
${ }^{1}$ Recall that the square of the $W^{1,2}$ norm of a map $f: \mathbf{S}^{1} \rightarrow \mathbf{R}^{N}$ is $\int_{\mathbf{S}^{1}}\left(|f|^{2}+\left|f^{\prime}\right|^{2}\right)$. Thus two curves that are $W^{1,2}$ close are also $C^{0}$ close; $\operatorname{cf}(1.4)$.
} 
(M2) The injectivity radius of $M$ is at least $8 \pi$ and the curvature is at most $1 / 64$, so that every geodesic ball of radius at most $4 \pi$ in $M$ is strictly geodesically convex;

(M3) If $x, y \in M$ with $|x-y| \leq 1$, then $\operatorname{dist}_{M}(x, y) \leq 2|x-y|$.

\subsection{The width}

Let $\Omega$ be the set of continuous maps $\sigma: \mathbf{S}^{1} \times[-1,1] \rightarrow M$ so that for each $t$ the map $\sigma(\cdot, t)$ is in $W^{1,2}$, the map $t \rightarrow \sigma(\cdot, t)$ is continuous from $[-1,1]$ to $W^{1,2}$, and finally $\sigma$ maps $\mathbf{S}^{1} \times\{-1\}$ and $\mathbf{S}^{1} \times\{1\}$ to points. Given a map $\hat{\sigma} \in \Omega$, the homotopy class $\Omega_{\widehat{\sigma}}$ is defined to be the set of maps $\sigma \in \Omega$ that are homotopic to $\hat{\sigma}$ through maps in $\Omega$. The width $W=W(\hat{\sigma})$ associated to the homotopy class $\Omega_{\widehat{\sigma}}$ is defined by taking inf of max of the energy of each slice. That is, set

$$
W=\inf _{\sigma \in \Omega_{\widehat{\sigma}}} \max _{t \in[-1,1]} \operatorname{Energy}(\sigma(\cdot, t)),
$$

where the energy is given by Energy $(\sigma(\cdot, t))=\int_{\mathbf{S}^{1}}\left|\partial_{x} \sigma(x, t)\right|^{2} d x$. The width is always nonnegative and is positive if $\hat{\sigma}$ is in a nontrivial homotopy class. ${ }^{2}$

The main theorem, Theorem 1.5, that almost maximal slices in the tightened sweepout are almost geodesics, is proven in Section 1.4. The proof of this theorem as well as the construction of the sequence of tighter and tighter sweepouts uses a curve shortening map that is defined in the next subsection. We also state the key properties of the shortening map in the next subsection, but postpone their proofs to Section 4 and the appendices.
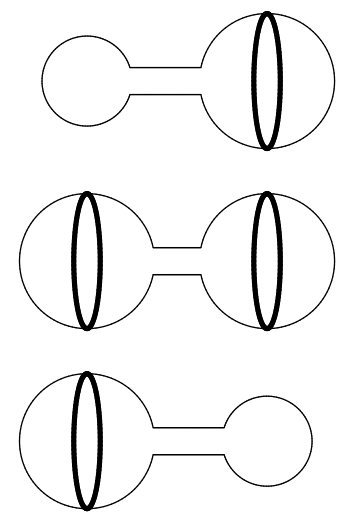

The width is continuous in the metric, but the min-max curve that realizes it may not be. In fact, elaborating on this example one can easily see that the width is not in general more than continuous in the metric.

The continuity of the width for a smooth oneparameter family of metrics $\left\{g_{t}\right\}_{t \in[0,1]}$ follows immediately from the following: Given $\epsilon>0$, there exists a $\delta>0$ such that if $t \in[0,1]$ and $|s-t|<\delta$, then $W\left(g_{s}\right)<W\left(g_{t}\right)+\epsilon$.

\footnotetext{
${ }^{2}$ A particularly interesting example is when $M$ is a topological 2 -sphere and the induced map from $\mathbf{S}^{2}$ to $M$ has degree one. In this case, the width is positive and realized by a nontrivial closed geodesic. To see that the width is positive on nontrivial homotopy classes, observe that if the maximal energy of a slice is sufficiently small, then each curve $\sigma(\cdot, t)$ is contained in a convex geodesic ball in $M$. Hence, a geodesic homotopy connects $\sigma$ to a path of point curves, so $\sigma$ is homotopically trivial.
} 


\subsection{Curve shortening $\Psi$}

Fix a large positive integer $L$ and let $\Lambda$ denote the space of piecewise linear maps from $\mathbf{S}^{1}$ to $M$ with exactly $L$ breaks (possibly with unnecessary breaks) such that the length of each geodesic segment is at most $2 \pi$, parametrized by a (constant) multiple of arclength, and with Lipschitz bound $L$. By a linear map, we mean a (constant speed) geodesic. Let $G \subset \Lambda$ denote the set of immersed closed geodesics in $M$ of length at most $2 \pi L$. (The energy of a curve in $\Lambda$ is equal to its length squared divided by $2 \pi$. In other words, energy and length are essentially equivalent.) Note that $\Lambda$ is finite dimensional with the dimension given in terms of $L$ and the dimension of $M$.

We will use the distance and topology on $\Lambda$ given by the $W^{1,2}$ norm on the space of maps from $\mathbf{S}^{1}$ to $M$.

The curve shortening is a map $\Psi: \Lambda \rightarrow \Lambda$ so that: ${ }^{3}$

(1) $\Psi(\gamma)$ is homotopic to $\gamma$ and Length $(\Psi(\gamma)) \leq \operatorname{Length}(\gamma)$.

(2) $\Psi(\gamma)$ depends continuously on $\gamma$.

(3) There is a continuous function $\phi:[0, \infty) \rightarrow[0, \infty)$ with $\phi(0)=0$ so that

$$
\operatorname{dist}^{2}(\gamma, \Psi(\gamma)) \leq \phi\left(\frac{\text { Length }^{2}(\gamma)-\text { Length }^{2}(\Psi(\gamma))}{\text { Length }^{2}(\Psi(\gamma))}\right) .
$$

(4) Given $\epsilon>0$, there exists $\delta>0$ so that if $\gamma \in \Lambda$ with $\operatorname{dist}(\gamma, G) \geq \epsilon$, then Length $(\Psi(\gamma)) \leq$ Length $(\gamma)-\delta$.

To define $\Psi$, we will fix a partition of $\mathbf{S}^{1}$ by choosing $2 L$ consecutive evenly spaced points ${ }^{4}$

$$
x_{0}, x_{1}, x_{2}, \ldots, x_{2 L}=x_{0} \in \mathbf{S}^{1},
$$

so that $\left|x_{j}-x_{j+1}\right|=\pi / L . \Psi(\gamma)$ is given in three steps. First, we apply Step 1 to $\gamma$ to get a curve $\gamma_{e}$, then we apply Step 2 to $\gamma_{e}$ to get a curve $\gamma_{o}$. In the third and final step, we reparametrize $\gamma_{o}$ to get $\Psi(\gamma)$.

Step 1 Replace $\gamma$ on each even interval, ie, $\left[x_{2 j}, x_{2 j+2}\right]$, by the linear map with the same endpoints to get a piecewise linear curve $\gamma_{e}: \mathbf{S}^{1} \rightarrow M$. Namely, for each $j$, we let $\left.\gamma_{e}\right|_{\left[x_{2 j}, x_{2 j+2}\right]}$ be the unique shortest (constant speed) geodesic from $\gamma\left(x_{2 j}\right)$ to $\gamma\left(x_{2 j+2}\right)$.

\footnotetext{
${ }^{3}$ This map is essentially what is usually called Birkhoff's curve shortening process; see Section 2 of [9].

${ }^{4}$ Note that this is not necessarily where the piecewise linear maps have breaks.
} 
Step 2 Replace $\gamma_{e}$ on each odd interval by the linear map with the same endpoints to get the piecewise linear curve $\gamma_{o}: \mathbf{S}^{1} \rightarrow M$.

Step 3 Reparametrize $\gamma_{o}$ (fixing $\gamma_{o}\left(x_{0}\right)$ ) to get the desired constant speed curve $\Psi(\gamma): \mathbf{S}^{1} \rightarrow M$.

It is easy to see that $\Psi$ maps $\Lambda$ to $\Lambda$ and has property (1); cf Section 2 of [9]. Properties (2), (3) and (4) for $\Psi$ are established in Section 4 and Appendix B. Throughout the rest of this section, we will assume these properties and use them to prove the main theorem.

The next lemma, which combines (3) and (4), is the key to producing the desired sequence of sweepouts.

Lemma 1.2 Given $W \geq 0$ and $\epsilon>0$, there exists $\delta>0$ so that if $\gamma \in \Lambda$ and

$$
2 \pi(W-\delta)<\operatorname{Length}^{2}(\Psi(\gamma)) \leq \text { Length }^{2}(\gamma)<2 \pi(W+\delta),
$$

then $\operatorname{dist}(\Psi(\gamma), G)<\epsilon$.

Proof If $W \leq \epsilon^{2} / 6$, the Wirtinger inequality ${ }^{5}$ gives the lemma with $\delta=\epsilon^{2} / 6$.

Assume next that $W>\epsilon^{2} / 6$. The triangle inequality gives

$$
\operatorname{dist}(\Psi(\gamma), G) \leq \operatorname{dist}(\Psi(\gamma), \gamma)+\operatorname{dist}(\gamma, G) .
$$

Since $\Psi$ does not decrease the length of $\gamma$ by much, property (4) of $\Psi$ allows us to bound $\operatorname{dist}(\gamma, G)$ by $\epsilon / 2$ as long as $\delta$ is sufficiently small. Similarly, property (3) of $\Psi$ allows us to bound $\operatorname{dist}(\Psi(\gamma), \gamma)$ by $\epsilon / 2$ as long as $\delta$ is sufficiently small.

\subsection{Defining the sweepouts}

Choose a sequence of maps $\hat{\sigma}^{j} \in \Omega_{\widehat{\sigma}}$ with

$$
\max _{t \in[-1,1]} \operatorname{Energy}\left(\hat{\sigma}^{j}(\cdot, t)\right)<W+\frac{1}{j} .
$$

Observe that (1.3) and the Cauchy-Schwarz inequality imply a uniform bound for the length and uniform $C^{1 / 2}$ continuity for the slices, that are both independent of $t$ and $j$.

${ }^{5}$ The Wirtinger inequality is just the usual Poincare inequality which bounds the $L^{2}$ norm in terms of the $L^{2}$ norm of the derivative; ie, $\int_{0}^{2 \pi} f^{2} d t \leq 4 \int_{0}^{2 \pi}\left(f^{\prime}\right)^{2} d t$ provided $f(0)=f(2 \pi)=0$. 
The first follows immediately and the latter follows from

$$
\begin{aligned}
\left|\hat{\sigma}^{j}(x, t)-\hat{\sigma}^{j}(y, t)\right|^{2} & \leq\left(\int_{x}^{y}\left|\partial_{s} \hat{\sigma}^{j}(s, t)\right| d s\right)^{2} \\
& \leq|y-x| \int_{x}^{y}\left|\partial_{s} \hat{\sigma}^{j}(s, t)\right|^{2} d s \leq|y-x|(W+1) .
\end{aligned}
$$

We will replace the $\hat{\sigma}^{j}$ 's by sweepouts $\sigma^{j}$ that, in addition to satisfying (1.3), also satisfy that the slices $\sigma^{j}(\cdot, t)$ are in $\Lambda$. We will do this by using local linear replacement similar to Step 1 of the construction of $\Psi$. Namely, the uniform $C^{1 / 2}$ bound for the slices allows us to fix a partition of points $y_{0}, \ldots, y_{N}=y_{0}$ in $\mathbf{S}^{1}$ so that each interval $\left[y_{i}, y_{i+1}\right]$ is always mapped to a ball in $M$ of radius at most $4 \pi$. Next, for each $t$ and each $j$, we replace $\left.\hat{\sigma}^{j}(\cdot, t)\right|_{\left[y_{i}, y_{i+1}\right]}$ by the linear map (geodesic) with the same endpoints and call the resulting map $\widetilde{\sigma}^{j}(\cdot, t)$. Reparametrize $\widetilde{\sigma}^{j}(\cdot, t)$ to have constant speed to get $\sigma^{j}(\cdot, t)$. It is easy to see that each $\sigma^{j}(\cdot, t)$ satisfies (1.3). Furthermore, the length bound for $\sigma^{j}(\cdot, t)$ also gives a uniform Lipschitz bound for the linear maps; let $L$ be the maximum of $N$ and this Lipschitz bound.

It remains to show that $\sigma^{j}$ is continuous in the transversal direction, ie, with respect to $t$, and homotopic to $\hat{\sigma}$ in $\Omega$. These facts were established already by Birkhoff [2; 3] (see also Section 2 of [9]), but also follow immediately from Appendix B.

Finally, applying the replacement map $\Psi$ to each $\sigma^{j}(\cdot, t)$ gives a new sequence of sweepouts $\gamma^{j}=\Psi\left(\sigma^{j}\right)$. (By Appendix B, $\Psi$ depends continuously on $t$ and preserves the homotopy class $\Omega_{\widehat{\sigma}}$; it is clear that $\Psi$ fixes the constant maps at $t= \pm 1$.)

\subsection{Almost maximal implies almost critical}

Our main result is that this sequence $\gamma^{j}$ of sweepouts is tight in the sense of the Introduction. Namely, we have the following theorem.

Theorem 1.5 Given $W \geq 0$ and $\epsilon>0$, there exist $\delta>0$ so that if $j>1 / \delta$ and for some $t_{0}$

$$
2 \pi \operatorname{Energy}\left(\gamma^{j}\left(\cdot, t_{0}\right)\right)=\operatorname{Length}^{2}\left(\gamma^{j}\left(\cdot, t_{0}\right)\right)>2 \pi(W-\delta),
$$

then for this $j$ we have dist $\left(\gamma^{j}\left(\cdot, t_{0}\right), G\right)<\epsilon$.

Proof Let $\delta$ be given by Lemma 1.2. By (1.6), (1.3), and using that $j>1 / \delta$, we get

$$
2 \pi(W-\delta)<\text { Length }^{2}\left(\gamma^{j}\left(\cdot, t_{0}\right)\right) \leq \text { Length }^{2}\left(\sigma^{j}\left(\cdot, t_{0}\right)\right)<2 \pi(W+\delta) .
$$

Thus, since $\gamma^{j}\left(\cdot, t_{0}\right)=\Psi\left(\sigma^{j}\left(\cdot, t_{0}\right)\right)$, Lemma $1.2 \operatorname{gives} \operatorname{dist}\left(\gamma^{j}\left(\cdot, t_{0}\right), G\right)<\epsilon$, as claimed. 


\subsection{Parameter spaces}

Instead of using the unit interval, $[0,1]$, as the parameter space for the circles in the sweepout and assuming that the curves start and end in point curves, we could have used any compact set $\mathcal{P}$ and required that the curves are constant on $\partial \mathcal{P}$ (or that $\partial \mathcal{P}=\varnothing$ ). In this case, let $\Omega^{\mathcal{P}}$ be the set of continuous maps $\sigma: \mathbf{S}^{1} \times \mathcal{P} \rightarrow M$ so that for each $t \in \mathcal{P}$ the curve $\sigma(\cdot, t)$ is in $W^{1,2}$, the map $t \rightarrow \sigma(\cdot, t)$ is continuous from $\mathcal{P}$ to $W^{1,2}$, and finally $\sigma$ maps $\partial \mathcal{P}$ to point curves. Given a map $\hat{\sigma} \in \Omega^{\mathcal{P}}$, the homotopy class $\Omega_{\widehat{\sigma}}^{\mathcal{P}} \subset \Omega^{\mathcal{P}}$ is defined to be the set of maps $\sigma \in \Omega^{\mathcal{P}}$ that are homotopic to $\hat{\sigma}$ through maps in $\Omega^{\mathcal{P}}$. Finally, the width $W=W(\hat{\sigma})$ is

$$
W=\inf _{\sigma \in \Omega_{\widehat{\sigma}}^{\mathcal{P}}} \max _{t \in \mathcal{P}} \operatorname{Energy}(\sigma(\cdot, t)) .
$$

Theorem 1.5 holds for these general parameter spaces; the proof is virtually the same with only trivial changes.

\section{Rate of change of width under mean curvature flow}

Recall that a one-parameter family of smooth hypersurfaces $\left\{M_{t}\right\} \subset \mathbf{R}^{n+1}$ with $n \geq 2$ flows by mean curvature if

$$
z_{t}=\mathbf{H}(z)=\Delta_{M_{t}} z,
$$

where $z$ are coordinates on $\mathbf{R}^{n+1}$ and $\mathbf{H}$ is the mean curvature vector. By Theorem 1.1 and Theorem 4.3 in Huisken [11], any smooth compact and strictly convex hypersurface in $\mathbf{R}^{n+1}$ remains smooth compact and strictly convex under the mean curvature flow until it disappears in a point. For such a hypersurface, the map which takes a point in $M$ to its unit normal gives a diffeomorphism from $M$ to $\mathbf{S}^{n}$. Since $\mathbf{S}^{n}=\{(x, y) \in$ $\left.\mathbf{R}^{2} \times\left.\mathbf{R}^{n-1}|| x\right|^{2}+|y|^{2}=1\right\}$ is equivalent to $\mathbf{S}^{1} \times \overline{B^{n-1}}$ where $B^{n-1}$ is the unit ball in $\mathbf{R}^{n-1}$ and $\mathbf{S}^{1} \times\{y\}$ for each $y \in \partial B^{n-1}$ is collapsed. In particular, we can fix a nontrivial homotopy class $\beta \in \Omega^{\overline{B^{n-1}}}$ in $\pi_{n}\left(M_{t}\right)$ and define the width $W(t)=W\left(\beta, M_{t}\right)$ using as parameter space $\mathcal{P}=\overline{B^{n-1}}$. It follows that the width $W(t)$ is positive for each $t$ up until the flow $M_{t}$ becomes extinct.

The next is the main result of this section. It applies Theorem 1.5 to bound the rate of change of the width $W(t)$ under the mean curvature flow.

Theorem 2.1 Let $\left\{M_{t}\right\}_{t \geq 0}$ be a one-parameter family of smooth compact and strictly convex hypersurfaces in $\mathbf{R}^{n+1}$ flowing by mean curvature, then in the sense of limsup 
of forward difference quotients

$$
\begin{aligned}
\frac{d}{d t} W & \leq-4 \pi, \\
W(t) & \leq W(0)-4 \pi t .
\end{aligned}
$$

If we have equality for $t=0$ in (2.2), then for $M_{0}$ the width is realized by a round circle in a plane. Moreover, on the circle in any direction tangent to $M_{0}$, but orthogonal to the circle, the second fundamental form vanishes. This follows from the cases of equality in the Cauchy-Schwarz inequality, the Borsuk-Fenchel inequality, and in (2.6) below.

As a consequence of Theorem 2.1, we get the following extinction result which is sharp in the case of shrinking cylinders, where the radius of the cylinders, $r(t)$, satisfies that $\frac{d}{d t} r^{2}=-2$, and, thus, $t_{\mathrm{ext}}=r^{2}(0) / 2=W(0) / 4 \pi$.

Corollary 2.4 Let $\left\{M_{t}\right\}_{t \geq 0}$ be a one-parameter family of smooth compact and strictly convex hypersurfaces in $\mathbf{R}^{n+1}$ flowing by mean curvature, then it becomes extinct after time at most

$$
\frac{W(0)}{4 \pi} .
$$

Although we have stated the results for compact convex hypersurfaces, the arguments apply to certain types of noncompact convex hypersurfaces; like shrinking cylinders. The main requirement is that the ends are "thin" so that the width is finite. We will not explore this here.

The key to proving the estimate on the rate of change of width is the following consequence of the first variation formula for volume (ie, 9.3 and $7.5^{\prime}$ in Simon [16]) and its corollary:

Lemma 2.5 Let $M_{t} \subset \mathbf{R}^{n+1}$ be smooth convex hypersurfaces that flow by mean curvature. If $\Sigma \subset M_{0}$ is a closed minimal submanifold and $\Sigma_{t}$ is the corresponding submanifold in $M_{t}$ with volume $V_{t}$, then

$$
\frac{d}{d t}{ }_{t=0} V_{t}=-\int_{\Sigma}\left\langle\mathbf{H}_{\Sigma}, \mathbf{H}_{M_{0}}\right\rangle \leq-\int_{\Sigma}\left|\mathbf{H}_{\Sigma}\right|^{2} .
$$

Here $\mathbf{H}_{\Sigma}$ is the mean curvature vector of $\Sigma$ as a submanifold of $\mathbf{R}^{n+1}$, which at $p \in \Sigma$ is equal to the trace of the second fundamental form $A_{M_{0}}$ restricted to $T_{p} \Sigma$ since $\Sigma$ is a minimal submanifold of $M_{0}$. 
Proof To get the inequality in (2.6) we used that since $\Sigma$ is a minimal submanifold of the convex hypersurface $M_{0} \subset \mathbf{R}^{n+1}$, then $\mathbf{H}_{\Sigma}$ points in the same direction as $\mathbf{H}_{M_{0}}$ and $\left|\mathbf{H}_{\Sigma}\right| \leq\left|\mathbf{H}_{M_{0}}\right|$.

In the first part of the next corollary, we will use the first variation formula for the energy asserting that if $\sigma_{t}:[0,2 \pi] \rightarrow \mathbf{R}^{n+1}$ is a one-parameter family of curves evolving by a vector field $\mathbf{V}$, then $\frac{d}{d t} \operatorname{Energy}\left(\sigma_{t}\right)=2 \int_{0}^{2 \pi}\left\langle\sigma_{t}^{\prime}, \nabla_{\sigma_{t}^{\prime}} \mathbf{V}\right\rangle$.

Corollary 2.7 Let $M_{t}, \Sigma, \Sigma_{t}, \mathbf{H}_{\Sigma}$, and $V_{t}$ be as in Lemma 2.5. If $\Sigma$ is a closed nonconstant geodesic parametrized on $\mathbf{S}^{1}$, then $V_{t}$ is the length of $\Sigma_{t}, \mathbf{H}_{\Sigma_{t}}$ its geodesic curvature as a curve in $\mathbf{R}^{n+1}$, and

$$
\begin{aligned}
\pi \frac{d}{d t}{ }_{t=0} \operatorname{Energy}\left(\Sigma_{t}\right)=V_{0} \frac{d}{d t} V_{t=0} & \leq-V_{0} \int_{\Sigma}\left|\mathbf{H}_{\Sigma}\right|^{2} \\
& \leq-\left(\int_{\Sigma}\left|\mathbf{H}_{\Sigma}\right|\right)^{2} \leq-4 \pi^{2} .
\end{aligned}
$$

If $\Sigma$ is a closed nonconstant minimal (2-dimensional) surface, then $V_{t}$ is the area of $\Sigma_{t}$ and

$$
\frac{d}{d t} V_{t=0} \leq-\int_{\Sigma}\left|\mathbf{H}_{\Sigma}\right|^{2} \leq-16 \pi
$$

Proof The first inequality in (2.8) follows from Lemma 2.5, the second from the Cauchy-Schwarz inequality, and the last inequality follows since by Borsuk-Fenchel's theorem every closed curve in $\mathbf{R}^{n+1}$ has total curvature at least $2 \pi$; see Borsuk [4] and Fenchel [10].

The first inequality in (2.9) follows from Lemma 2.5. The second inequality is (1.4) in [17], but we include the proof. Namely, use $\Delta_{\Sigma}|z|^{2}=4+2\left\langle z, \mathbf{H}_{\Sigma}\right\rangle$ and $\left.\left.\left|\nabla_{\Sigma}\right| z\right|^{2}\right|^{2}=$ $4\left(|z|^{2}-\left|z^{\perp}\right|^{2}\right)$ to compute

$$
\Delta_{\Sigma} \log |z|^{2}=2 \frac{\left\langle z, \mathbf{H}_{\Sigma}\right\rangle}{|z|^{2}}+4 \frac{\left|z^{\perp}\right|^{2}}{|z|^{4}}=\left|\frac{1}{2} \mathbf{H}_{\Sigma}+2 \frac{z^{\perp}}{|z|^{2}}\right|^{2}-\frac{1}{4}\left|\mathbf{H}_{\Sigma}\right|^{2},
$$

where $z$ is the position vector in $\mathbf{R}^{n+1}$, and $z^{\perp}$ is the projection of $z$ to the normal space of $\Sigma$ at the point $z$. Applying Stokes' theorem to $-\Delta_{\Sigma} \log |z|^{2}$ gives

$$
\lim _{r \rightarrow 0} \frac{\left.\int_{\partial B_{r} \cap \Sigma}\left|\nabla_{\Sigma}\right| z\right|^{2} \mid}{r^{2}} \leq \frac{1}{4} \int_{\Sigma}\left|\mathbf{H}_{\Sigma}\right|^{2} .
$$

Here $B_{r}$ is the ball of radius $r$ about 0 in $\mathbf{R}^{n+1}$. Since $\int_{\Sigma}\left|\mathbf{H}_{\Sigma}\right|^{2}$ is translation invariant, we can translate so that $0 \in \Sigma$ and, thus, $\left.\lim _{r \rightarrow 0} r^{-2} \int_{\partial B_{r} \cap \Sigma}\left|\nabla_{\Sigma}\right| z\right|^{2} \mid$ is at least $4 \pi$. 
The last ingredient needed in the proof of Theorem 2.1 is the following consequence of the first variation formula for the energy: If $\mathbf{V}$ is a $C^{2}$ vector field and $\sigma_{t}, \eta_{t}$ are in $W^{1,2}$, then

(2.10) $\left|\frac{d}{d t} \operatorname{Energy}\left(\eta_{t}\right)-\frac{d}{d t} \operatorname{Energy}\left(\sigma_{t}\right)\right| \leq C\|\mathbf{V}\|_{C^{2}}\left\|\sigma_{t}-\eta_{t}\right\|_{W^{1,2}}\left(1+\sup \left|\sigma_{t}^{\prime}\right|^{2}\right)$.

Proof of of Theorem 2.1 Fix a time $\tau$. Below $C$ denotes a constant depending only on $M_{\tau}$ but will be allowed to change from inequality to inequality. Let $\gamma^{j}$ be the sequence of sweepouts in $M_{\tau}$ defined in Section 1.3. In particular, the maximal energy of a slice in $\gamma^{j}$ goes to $W(\tau)$ as $j \rightarrow \infty$, the $\gamma^{j}$ 's are "tightened" in the sense of Theorem 1.5, and $\gamma_{s}^{j}$ has Lipschitz bound $L$ independent of $j$ and $s$. For $t \geq \tau$, let $\sigma_{s}^{j}(t)$ be the curve in $M_{t}$ that corresponds to $\gamma_{s}^{j}$ and set $e_{s, j}(t)=\operatorname{Energy}\left(\sigma_{s}^{j}(t)\right)$. We will use $\sigma_{s}^{j}(t)$ as a comparison to get an upper bound for the width at times $t>\tau$. The key for this is the following claim: Given $\epsilon>0$, there exist $\delta>0$ and $h_{0}>0$ so that if $j>1 / \delta$ and $0<h<h_{0}$, then for all $s \in \mathcal{P}$

$$
e_{s, j}(\tau+h)-\max _{s_{0}} e_{s_{0}, j}(\tau) \leq[-4 \pi+C \epsilon] h+C h^{2} .
$$

To see why (2.11) implies (2.2), take the limit as $j \rightarrow \infty$ (so that $\max _{s_{0}} e_{s_{0}, j}(\tau) \rightarrow$ $W(\tau))$ in (2.11) to get

$$
\frac{W(\tau+h)-W(\tau)}{h} \leq-4 \pi+C \epsilon+C h .
$$

Taking $\epsilon \rightarrow 0$ in (2.12) gives (2.2).

It remains to prove (2.11). First, let $\delta>0$, depending on $\epsilon$ (and on $\tau$ ), be given by Theorem 1.5. Since $\beta$ is nontrivial in $\pi_{n}\left(M_{\tau}\right), W(\tau)$ is positive and, so, we can assume that $\epsilon^{2}<W(\tau) / 3$ and $\delta<W(\tau) / 3$. If $j>1 / \delta$ and $e_{s, j}(\tau)>W(\tau)-\delta$, then Theorem 1.5 gives a nonconstant closed geodesic $\eta$ in $M_{\tau}$ with $\operatorname{dist}\left(\eta, \gamma_{s}^{j}\right)<\epsilon$. As in Lemma 2.5, let $\eta_{t}$ denote the image of $\eta$ in $M_{t}$. Combining (2.8) and (2.10) with $\mathbf{V}=\mathbf{H}_{M_{t}}$ and using the uniform Lipschitz bound $L$ for the sweepouts at time $\tau$ gives

(2.13) $\frac{d}{d t} e_{t=\tau} e_{s, j}(t) \leq \frac{d}{d t} \underset{t=\tau}{\operatorname{Energy}}\left(\eta_{t}\right)+C \epsilon\left\|\mathbf{H}_{M_{\tau}}\right\|_{C^{2}}\left(1+L^{2}\right) \leq-4 \pi+C \epsilon$.

Since $\sigma_{s}^{j}(t)$ is the composition of $\gamma_{s}^{j}$ with the smooth flow and $\gamma_{s}^{j}$ has Lipschitz bound $L$ independent of $j$ and $s$, it is easy to see that $e_{s, j}(\tau+h)$ is a smooth function of $h$ with a uniform $C^{2}$ bound independent of both $j$ and $s$ near $h=0$. In particular, (2.13) and Taylor expansion gives $h_{0}>0$ (independent of $j$ ) so that (2.11) holds for $s$ with $e_{s, j}(\tau)>W(\tau)-\delta$. In the remaining case, we have $e_{s, j}(\tau) \leq W(\tau)-\delta$ so the continuity of $W(t)$ implies that (2.11) automatically holds after possibly shrinking $h_{0}>0$. 
To get (2.3), observe that for any $\epsilon>0$ the set $\{t \mid W(t) \leq W(0)-(4 \pi-\epsilon) t\}$ contains 0 , is closed since $W(t)$ is continuous. By (2.2), it is also open. Therefore, $W(t) \leq$ $W(0)-(4 \pi-\epsilon) t$ for all $t$ up to the extinction time; taking $\epsilon \rightarrow 0$ gives (2.3).

\subsection{2-Width}

Instead of defining the width by using sweepouts by closed curves, we can define the width, $W_{2},(2-$-width) by sweeping out the manifold by 2 -spheres, the width being the min-max value of the energies ${ }^{6}$ or, equivalently, the areas of the slices in the sweepout. In $[7 ; 8]$ we defined the width in this way. Using (2.9) in place of (2.8) and arguing much like above (cf also with $[7 ; 8]$ ) we get the following (and the corresponding extinction estimate; cf Corollary 2.4):

Theorem 2.14 Let $\left\{M_{t}\right\}_{t \geq 0}$ be a one-parameter family of smooth compact and strictly convex hypersurfaces in $\mathbf{R}^{n+1}$ flowing by mean curvature, then in the sense of limsup of forward difference quotients

$$
\begin{aligned}
& \frac{d}{d t} W_{2} \leq-16 \pi, \\
& W_{2}(t) \leq W_{2}(0)-16 \pi t .
\end{aligned}
$$

\section{Evolution by powers of mean curvature}

Suppose that $k>0$ and a one-parameter family of smooth hypersurfaces $\left\{M_{t}\right\} \subset \mathbf{R}^{n+1}$ with $n \geq 2$ flows by

$$
z_{t}=|\mathbf{H}(z)|^{k} \mathbf{n}(z)=\left|\Delta_{M_{t}}(z)\right|^{k} \mathbf{n}(z),
$$

where $z$ are coordinates on $\mathbf{R}^{n+1}, \mathbf{n}=\mathbf{H}(z) /|\mathbf{H}(z)|$ is the unit normal, and $\mathbf{H}$ is the mean curvature vector.

In Theorem 1.1 of [15], F Schulze extended Huisken's result to evolution by any positive power of mean curvature. Namely, if $M_{0}$ is compact, smooth, and strictly convex, then the flow (3.1) is smooth and remains convex until it becomes extinct.

Theorem 2.1 and its corollary have analogs for these more general flows. Namely, we get a differential inequality for the width,

$$
\frac{1}{1+k} \frac{d}{d t} W_{t=0}^{k+1} \leq-(2 \pi)^{(k+1) / 2},
$$

${ }^{6}$ The energy of a map $u: \mathbf{S}^{2} \rightarrow \mathbf{R}^{n+1}$ is $\frac{1}{2} \int_{\mathbf{S}^{2}}|\nabla u|^{2}$. 
that implies extinction in finite time. The proof relies on versions of Lemma 2.5 and Corollary 2.7 that are stated below. The proofs of these are virtually the same as those in Section 2 with the obvious changes. In particular, we use Hölder's inequality in Corollary 3.3 instead of Cauchy-Schwarz.

Lemma 3.2 Let $M_{t} \subset \mathbf{R}^{n+1}$ be smooth convex hypersurfaces that flow by (3.1). If $\Sigma \subset M_{0}$ is a closed minimal submanifold and $\Sigma_{t}$ is the corresponding submanifold in $M_{t}$ with volume $V_{t}$, then

$$
\frac{d}{d t}{ }_{t=0} V_{t}=-\int_{\Sigma}\left\langle\mathbf{H}_{\Sigma},\left|\mathbf{H}_{M_{0}}\right|^{k} \mathbf{n}_{M_{0}}\right\rangle \leq-\int_{\Sigma}\left|\mathbf{H}_{\Sigma}\right|^{1+k} .
$$

Corollary 3.3 Let $M_{t}, \Sigma, \Sigma_{t}, \mathbf{H}_{\Sigma}$, and $V_{t}$ be as in Lemma 2.5. If $\Sigma$ is a closed nonconstant geodesic parametrized on $\mathbf{S}^{1}$, then $V_{t}$ is the length of $\Sigma_{t}, \mathbf{H}_{\Sigma_{t}}$ its geodesic curvature as a curve in $\mathbf{R}^{n+1}$, and

$$
\begin{aligned}
\frac{1}{1+k} \frac{d}{d t}{ }_{t=0} V_{t}^{k+1}=V_{0}^{k} \frac{d}{d t}{ }_{t=0} V_{t} & \leq-V_{0}^{k} \int_{\Sigma}\left|\mathbf{H}_{\Sigma}\right|^{1+k} \\
& \leq-\left(\int_{\Sigma}\left|\mathbf{H}_{\Sigma}\right|\right)^{k+1} \leq-(2 \pi)^{k+1} .
\end{aligned}
$$

\section{Establishing Properties (2), (3) and (4) for $\Psi$}

To prove (2) and (3), it is useful to observe that there is an equivalent, but more symmetric, way to construct $\Psi(\gamma)$ using four steps:

$\left(\mathrm{A}_{1}\right)$ Follow Step 1 to get $\gamma_{e}$.

$\left(\mathrm{B}_{1}\right)$ Reparametrize $\gamma_{e}$ (fixing the image of $x_{0}$ ) to get the constant speed curve $\tilde{\gamma}_{e}$. This reparametrization moves the points $x_{j}$ to new points $\tilde{x}_{j}$ (ie, $\gamma_{e}\left(x_{j}\right)=$ $\left.\tilde{\gamma}_{e}\left(\tilde{x}_{j}\right)\right)$.

$\left(\mathrm{A}_{2}\right)$ Do linear replacement on the odd $\tilde{x}_{j}$ intervals to get $\tilde{\gamma}_{o}$.

$\left(\mathrm{B}_{2}\right)$ Reparametrize $\tilde{\gamma}_{o}$ (fixing the image of $\left.x_{0}\right)$ to get the constant speed curve $\Psi(\gamma)$.

The reason that this gives the same curve is that $\tilde{\gamma}_{o}$ is just a reparametrization of $\gamma_{o}$. We will also use that each of the four steps is energy nonincreasing. This is obvious for the linear replacements, since linear maps minimize energy. It follows from the Cauchy-Schwarz inequality for the reparametrizations, since for a curve $\sigma: \mathbf{S}^{1} \rightarrow M$ we have

$$
\operatorname{Length}^{2}(\sigma) \leq 2 \pi \operatorname{Energy}(\sigma)
$$


with equality if and only if $\left|\sigma^{\prime}\right|=\operatorname{Length}(\sigma) /(2 \pi)$ almost everywhere.

Using the alternative way of defining $\Psi(\gamma)$ in four steps, we see that (3) follows from the triangle inequality once we bound $\operatorname{dist}\left(\gamma, \gamma_{e}\right)$ and $\operatorname{dist}\left(\gamma_{e}, \tilde{\gamma}_{e}\right)$ in terms of the decrease in length (as well as the analogs for Steps $\left(\mathrm{A}_{2}\right)$ and $\left(\mathrm{B}_{2}\right)$ ).

The bound on $\operatorname{dist}\left(\gamma, \gamma_{e}\right)$ follows directly from the following; see Appendix A for the proof:

Lemma 4.1 There exists $C$ so that if $I$ is an interval of length at most $2 \pi / L, \sigma_{1}: I \rightarrow$ $M$ is a Lipschitz curve with $\left|\sigma_{1}^{\prime}\right| \leq L$, and $\sigma_{2}: I \rightarrow M$ is the minimizing geodesic with the same endpoints, then

$$
\operatorname{dist}^{2}\left(\sigma_{1}, \sigma_{2}\right) \leq C\left(\operatorname{Energy}\left(\sigma_{1}\right)-\operatorname{Energy}\left(\sigma_{2}\right)\right) .
$$

Applying Lemma 4.1 on each of the $L$ intervals in Step $\left(\mathrm{A}_{1}\right)$, we get that $\operatorname{dist}^{2}\left(\gamma, \gamma_{e}\right) \leq C\left(\operatorname{Energy}(\gamma)-\operatorname{Energy}\left(\gamma_{e}\right)\right) \leq \frac{C}{2 \pi}\left(\operatorname{Length}^{2}(\gamma)-\operatorname{Length}^{2}(\Psi(\gamma))\right)$. This gives the desired bound on $\operatorname{dist}\left(\gamma, \gamma_{e}\right) \operatorname{since} \operatorname{Length}(\Psi(\gamma)) \leq 2 \pi L$.

In bounding $\operatorname{dist}\left(\gamma_{e}, \tilde{\gamma}_{e}\right)$, we will use that $\gamma_{e}$ is just the composition $\tilde{\gamma}_{e} \circ P$, where $P: \mathbf{S}^{1} \rightarrow \mathbf{S}^{1}$ is a monotone piecewise linear map. ${ }^{7}$ Using that $\left|\tilde{\gamma}_{e}^{\prime}\right|=\operatorname{Length}\left(\tilde{\gamma}_{e}\right) /(2 \pi)$ (away from the breaks) and that the integral of $P^{\prime}$ is $2 \pi$, an easy calculation gives

$$
\begin{aligned}
\int\left(P^{\prime}-1\right)^{2} & =\int\left(P^{\prime}\right)^{2}-2 \pi=\int\left(\frac{\left|\gamma_{e}^{\prime}\right|}{\left|\widetilde{\gamma}_{e}^{\prime} \circ P\right|}\right)^{2}-2 \pi=\frac{4 \pi^{2}}{\operatorname{Length}^{2}\left(\tilde{\gamma}_{e}\right)} \int\left|\gamma_{e}^{\prime}\right|^{2}-2 \pi \\
\text { (4.2) } & =2 \pi \frac{\operatorname{Energy}\left(\gamma_{e}\right)-\operatorname{Energy}\left(\tilde{\gamma}_{e}\right)}{\operatorname{Energy}\left(\tilde{\gamma}_{e}\right)} \leq 2 \pi \frac{\operatorname{Energy}(\gamma)-\operatorname{Energy}(\Psi(\gamma))}{\operatorname{Energy}(\Psi(\gamma))} .
\end{aligned}
$$

Since $\gamma_{e}$ and $\tilde{\gamma}_{e}$ agree at $x_{0}=x_{2 L}$, the Wirtinger inequality (footnote 5) bounds $\operatorname{dist}^{2}\left(\gamma_{e}, \tilde{\gamma}_{e}\right)$ in terms of

$$
\int\left|\left(\tilde{\gamma}_{e} \circ P\right)^{\prime}-\tilde{\gamma}_{e}^{\prime}\right|^{2} \leq 2 \int\left|\left(\tilde{\gamma}_{e}^{\prime} \circ P\right) P^{\prime}-\tilde{\gamma}_{e}^{\prime} \circ P\right|^{2}+2 \int\left|\tilde{\gamma}_{e}^{\prime} \circ P-\tilde{\gamma}_{e}^{\prime}\right|^{2} .
$$

We will bound both terms on the right hand side of (4.3) in terms of $\int\left|P^{\prime}-1\right|^{2}$ and then appeal to (4.2). To bound the first term, use that $\left|\tilde{\gamma}_{e}^{\prime}\right|$ is (a constant) $\leq L$ to get

$$
\int\left|\left(\tilde{\gamma}_{e}^{\prime} \circ P\right) P^{\prime}-\tilde{\gamma}_{e}^{\prime} \circ P\right|^{2} \leq L^{2} \int\left|P^{\prime}-1\right|^{2} .
$$

To bound the second integral, we will use that when $x$ and $y$ are points in $\mathbf{S}^{1}$ that are not separated by a break point, then $\tilde{\gamma}_{e}$ is a geodesic from $x$ to $y$ and, thus, $\tilde{\gamma}_{e}^{\prime \prime}$ is

\footnotetext{
${ }^{7}$ The map $P$ is Lipschitz, but the inverse map $P^{-1}$ may not be if $\gamma_{e}$ is constant on an interval.
} 
normal to $M$ and $\left|\tilde{\gamma}_{e}^{\prime \prime}\right| \leq\left|\tilde{\gamma}_{e}^{\prime}\right|^{2} \sup _{M}|A| \leq L^{2} / 16$. Therefore, integrating $\tilde{\gamma}_{e}^{\prime \prime}$ from $x$ to $y$ gives

$$
\left|\tilde{\gamma}_{e}^{\prime}(x)-\tilde{\gamma}_{e}^{\prime}(y)\right| \leq|x-y| \sup \left|\tilde{\gamma}_{e}^{\prime \prime}\right| \leq \frac{L^{2}}{16}|x-y| .
$$

Divide $\mathbf{S}^{1}$ into two sets, $S_{1}$ and $S_{2}$, where $S_{1}$ is the set of points within distance $\left(\pi \int\left|P^{\prime}-1\right|^{2}\right)^{1 / 2}$ of a break point for $\tilde{\gamma}_{e}$. Since $P\left(x_{0}\right)=x_{0}$, arguing as in (1.4) gives $|P(x)-x| \leq\left(\pi \int\left|P^{\prime}-1\right|^{2}\right)^{1 / 2}$. Thus, if $x \in S_{2}$, then $\tilde{\gamma}_{e}$ is smooth between $x$ and $P(x)$. Consequently, (4.4) gives

$$
\int_{S_{2}}\left|\tilde{\gamma}_{e}^{\prime} \circ P-\tilde{\gamma}_{e}^{\prime}\right|^{2} \leq \frac{L^{4}}{256} \int_{S_{2}}|P(s)-s|^{2} \leq \frac{L^{4}}{64} \int\left|P^{\prime}-1\right|^{2},
$$

where the last inequality used the Wirtinger inequality. On the other hand,

$$
\int_{S_{1}}\left|\tilde{\gamma}_{e}^{\prime} \circ P-\tilde{\gamma}_{e}^{\prime}\right|^{2} \leq 4 L^{2} \operatorname{Length}\left(S_{1}\right) \leq 8 L^{3}\left(\pi \int\left|P^{\prime}-1\right|^{2}\right)^{1 / 2},
$$

completing the proof of property (3).

We show (2) in Appendix B.

To prove property (4), we will argue by contradiction. Suppose therefore that there exist $\epsilon>0$ and a sequence $\gamma_{j} \in \Lambda$ with $\operatorname{Energy}\left(\Psi\left(\gamma_{j}\right)\right) \geq \operatorname{Energy}\left(\gamma_{j}\right)-1 / j$ and $\operatorname{dist}\left(\gamma_{j}, G\right) \geq \epsilon>0$; note that the second condition implies a positive lower bound for $\operatorname{Energy}\left(\gamma_{j}\right)$. Observe next that the space $\Lambda$ is compact ${ }^{8}$ and, thus, a subsequence of the $\gamma_{j}$ 's must converge to some $\gamma \in \Lambda$. Since property (3) implies that $\operatorname{dist}\left(\gamma_{j}, \Psi\left(\gamma_{j}\right)\right) \rightarrow 0$, the $\Psi\left(\gamma_{j}\right)$ 's also converge to $\gamma$. The continuity of $\Psi$, ie, property (2) of $\Psi$, then implies that $\Psi(\gamma)=\gamma$. However, this implies that $\gamma \in G$ since the only fixed points of $\Psi$ are immersed closed geodesics. This last fact, which was used already by Birkhoff (see Section 2 in [9]), follows immediately from Lemma 4.1 and (4.2). However, this would contradict that the $\gamma_{j}$ 's remain a fixed distance from any such closed immersed geodesic, completing the proof of (4).

\section{Appendix A Proof of Lemma 4.1}

We will need a simple consequence of (M1) and (M3) in Section 1.

Lemma A.1 If $x, y \in M$, then $\left|(x-y)^{\perp}\right| \leq|x-y|^{2}$, where $(x-y)^{\perp}$ is the normal component to $M$ at $y$.

\footnotetext{
${ }^{8}$ Compactness of $\Lambda$ follows since $\sigma \in \Lambda$ depends continuously on the images of the $L$ break points in the compact manifold $M$.
} 
Proof If $|x-y| \geq 1$, then the claim is clear. Assume therefore that $|x-y|<1$ and $\alpha:[0, \ell] \rightarrow M$ is a minimizing unit speed geodesic from $y$ to $x$ with $\ell \leq 2|x-y|$. Let $V$ be the unit normal vector $V=(x-y)^{\perp} /\left|(x-y)^{\perp}\right|$, so $\left\langle\alpha^{\prime}(0), V\right\rangle=0$, and observe that

$$
\begin{aligned}
\left|(x-y)^{\perp}\right| & =\int_{0}^{\ell}\left\langle\alpha^{\prime}(s), V\right\rangle d s \\
& =\int_{0}^{\ell}\left\langle\alpha^{\prime}(0)+\int_{0}^{s} \alpha^{\prime \prime}(t) d t, V\right\rangle d s \leq \int_{0}^{\ell} \int_{0}^{s}\left|\alpha^{\prime \prime}(t)\right| d t d s \\
& \leq \int_{0}^{\ell} \int_{0}^{s}|A(\alpha(t))| d t d s \leq \frac{1}{2} \ell^{2} \sup _{M}|A| \leq|x-y|^{2} .
\end{aligned}
$$

Proof (of Lemma 4.1). Integrating by parts and using that $\sigma_{1}$ and $\sigma_{2}$ are equal on $\partial I$ gives

$$
\int_{I}\left|\sigma_{1}^{\prime}\right|^{2}-\int_{I}\left|\sigma_{2}^{\prime}\right|^{2}-\int_{I}\left|\left(\sigma_{1}-\sigma_{2}\right)^{\prime}\right|^{2}=-2 \int_{I}\left\langle\left(\sigma_{1}-\sigma_{2}\right), \sigma_{2}^{\prime \prime}\right\rangle \equiv \kappa .
$$

The lemma will follow by bounding $|\kappa|$ by $\frac{1}{2} \int_{I}\left|\left(\sigma_{1}-\sigma_{2}\right)^{\prime}\right|^{2}$ and appealing to Wirtinger's inequality.

Since $\sigma_{2}$ is a geodesic on $M, \sigma_{2}^{\prime \prime}$ is normal to $M$ and $\left|\sigma_{2}^{\prime \prime}\right| \leq\left|\sigma_{2}^{\prime}\right|^{2} \sup _{M}|A| \leq$ $\left|\sigma_{2}^{\prime}\right|^{2} / 16$. Thus, Lemma A.1 gives

$$
\left|\left\langle\left(\sigma_{1}-\sigma_{2}\right), \sigma_{2}^{\prime \prime}\right\rangle\right| \leq\left|\left(\sigma_{1}-\sigma_{2}\right)^{\perp}\right| \frac{\left|\sigma_{2}^{\prime}\right|^{2}}{16} \leq\left|\sigma_{1}-\sigma_{2}\right|^{2} \frac{\left|\sigma_{2}^{\prime}\right|^{2}}{16} .
$$

Integrating (A.2), using that $\left|\sigma_{2}^{\prime}\right|$ is constant with $\left|\sigma_{2}^{\prime}\right| \operatorname{Length}(I) \leq 2 \pi$, and applying Wirtinger's inequality gives

$$
\begin{aligned}
|\kappa| \leq \frac{\left|\sigma_{2}^{\prime}\right|^{2}}{8} \int_{I}\left|\sigma_{1}-\sigma_{2}\right|^{2} & \leq \frac{\left|\sigma_{2}^{\prime}\right|^{2}}{8}\left(\frac{\operatorname{Length}(I)}{\pi}\right)^{2} \int_{I}\left|\left(\sigma_{1}-\sigma_{2}\right)^{\prime}\right|^{2} \\
& \leq \frac{1}{2} \int_{I}\left|\left(\sigma_{1}-\sigma_{2}\right)^{\prime}\right|^{2} .
\end{aligned}
$$

\section{Appendix B The continuity of $\Psi$}

Lemma B.1 Let $\gamma: \mathbf{S}^{1} \rightarrow M$ be a $W^{1,2}$ map with $\operatorname{Energy}(\gamma) \leq L$. If $\gamma_{e}$ and $\tilde{\gamma}_{e}$ are given by applying Steps $\left(A_{1}\right)$ and $\left(B_{1}\right)$ to $\gamma,{ }^{9}$ then the map $\gamma \rightarrow \tilde{\gamma}_{e}$ is continuous from $W^{1,2}$ to $\Lambda$ equipped with the $W^{1,2}$ norm.

\footnotetext{
${ }^{9}\left(\mathrm{~A}_{1}\right)$ and $\left(\mathrm{B}_{1}\right)$ are defined in the beginning of Section 4.
} 
Proof It follows from (1.4) and the energy bound that $\operatorname{dist}_{M}\left(\gamma\left(x_{2 j}\right), \gamma\left(x_{2 j+2}\right)\right) \leq 2 \pi$ for each $j$ and thus we can apply Step $\left(\mathrm{A}_{1}\right)$. The lemma will follow easily from two observations:

(C1) Since $W^{1,2}$ close curves are also $C^{0}$ close (cf footnote 1), it follows that the points $\gamma_{e}\left(x_{2 j}\right)=\gamma\left(x_{2 j}\right)$ are continuous with respect to the $W^{1,2}$ norm.

(C2) Define $\Gamma \subset M \times M$ by $\Gamma=\left\{(x, y) \in M \times M \mid \operatorname{dist}_{M}(x, y) \leq 4 \pi\right\}$, and define a map $H: \Gamma \rightarrow C^{1}([0,1], M)$ by letting $H(x, y):[0,1] \rightarrow M$ be the linear map from $x$ to $y$. Then the map $H$ is continuous on $\Gamma$. Furthermore, the map $t \rightarrow H(x, y)(t)$ has uniformly bounded first and second derivatives $\left|\partial_{t} H(x, y)\right| \leq 4 \pi$ and $\left|\partial_{t}^{2} H(x, y)\right| \leq \pi^{2}$; the second derivative bound comes from (M1). ${ }^{10}$

To prove the lemma, suppose that $\gamma^{1}$ and $\gamma^{2}$ are nonconstant curves in $\Lambda$ (continuity at the constant maps is obvious). For $i=1,2$ and $j=1, \ldots, L$, let $a_{j}^{i}$ be the distance in $M$ from $\gamma^{i}\left(x_{2 j}\right)$ to $\gamma^{i}\left(x_{2 j+2}\right)$. Let $S^{i}=(1 / 2 \pi) \sum_{j=1}^{L} a_{j}^{i}$ be the speed of $\tilde{\gamma}_{e}^{i}$, so that $\left|\left(\widetilde{\gamma}_{e}^{i}\right)^{\prime}\right|=S^{i}$ except at the $L$ break points. By (C1), the $a_{j}^{i}$ 's are continuous functions of $\gamma^{i}$ and, thus, so are $S^{1}$ and $S^{2}$. Moreover, (C1) and (C2) imply that $\gamma_{e}^{1}$ and $\gamma_{e}^{2}$ are $C^{1}$-close on each interval $\left[x_{2 j}, x_{2 j+2}\right]$. Thus, we have shown that $\gamma \rightarrow \gamma_{e}$ is continuous.

To show that $\gamma_{e} \rightarrow \tilde{\gamma}_{e}$ is also continuous, we will show that the $\tilde{\gamma}_{e}^{i}$ 's are close when the $\gamma_{e}^{i}$ 's are. Since the point $x_{0}=x_{2 L}$ is fixed under the reparametrization, this will follow from applying Wirtinger's inequality to $\left(\tilde{\gamma}_{e}^{1}-\tilde{\gamma}_{e}^{2}\right)-\left(\tilde{\gamma}_{e}^{1}-\tilde{\gamma}_{e}^{2}\right)\left(x_{0}\right)$ once we show that $\int_{\mathbf{S}^{1}}\left|\left(\tilde{\gamma}_{e}^{1}-\tilde{\gamma}_{e}^{2}\right)^{\prime}\right|^{2}$ can be made small.

The piecewise linear curve $\tilde{\gamma}_{e}^{i}$ is linear on the intervals

$$
I_{j}^{i}=\left[\frac{1}{S^{i}} \sum_{\ell<j} a_{\ell}^{i}, \frac{1}{S^{i}} \sum_{\ell \leq j} a_{\ell}^{i}\right] .
$$

Set $I_{j}=I_{j}^{1} \cap I_{j}^{2}$. Observe first that since the intervals $I_{j}^{i}$ in (B.2) depend continuously on $\gamma_{e}^{i}$, the measure of the complement $\mathbf{S}^{1} \backslash\left[\bigcup_{j=1}^{L} I_{j}\right]$ can be made small, so that

$$
\int_{\mathbf{S}^{1} \backslash\left[\cup I_{j}\right]}\left|\left(\tilde{\gamma}_{e}^{1}-\tilde{\gamma}_{e}^{2}\right)^{\prime}\right|^{2} \leq 4 L^{2} \text { Length }\left(\mathbf{S}^{1} \backslash\left[\cup I_{j}\right]\right)
$$

can also be made small. We will divide the $I_{j}$ 's into two groups, depending on the size of $a_{j}^{1}$. Fix some $\epsilon>0$ and suppose first that $a_{j}^{1}<\epsilon$; by continuity, we can assume

${ }^{10}$ (M1) is defined in the beginning of Section 1. 
that $a_{j}^{2}<2 \epsilon$. For such a $j$, we get

$$
\int_{I_{j}}\left|\left(\tilde{\gamma}_{e}^{1}-\tilde{\gamma}_{e}^{2}\right)^{\prime}\right|^{2} \leq 2 \int_{I_{j}^{1}}\left|\left(\tilde{\gamma}_{e}^{1}\right)^{\prime}\right|^{2}+2 \int_{I_{j}^{2}}\left|\left(\tilde{\gamma}_{e}^{2}\right)^{\prime}\right|^{2} \leq 2 L\left(a_{j}^{1}+a_{j}^{2}\right) \leq 6 \epsilon L .
$$

Since there are at most $L$ breaks, summing over these intervals contributes at most $6 \epsilon L^{2}$ to the energy of $\left(\tilde{\gamma}_{e}^{1}-\tilde{\gamma}_{e}^{2}\right)$.

The last case to consider is an $I_{j}$ with $a_{j}^{1} \geq \epsilon$; by continuity, we can assume that $a_{j}^{2} \geq \epsilon / 2$. In this case, $\tilde{\gamma}_{e}^{i}$ can be written on $I_{j}$ as the composition $\gamma_{e}^{i} \circ P_{j}^{i}$ where $\left|\left(P_{j}^{i}\right)^{\prime}\right|=2 \pi S^{i} /\left(L a_{j}^{i}\right)$. Furthermore, $P_{j}^{1}$ and $P_{j}^{2}$ both map $I_{j}$ into $\left[x_{2 j}, x_{2 j+2}\right]$ and

$$
\int_{I_{j}}\left|\left(\tilde{\gamma}_{e}^{1}-\tilde{\gamma}_{e}^{2}\right)^{\prime}\right|^{2}=\int_{I_{j}}\left|\left(\gamma_{e}^{1} \circ P_{j}^{1}-\gamma_{e}^{2} \circ P_{j}^{2}\right)^{\prime}\right|^{2} .
$$

Finally, this can be made small since the speed $\left|\left(P_{j}^{i}\right)^{\prime}\right|$ is continuous ${ }^{11}$ in $\gamma^{i}$ and the $\gamma_{e}^{i}$,s are $C^{2}$ bounded and $C^{1}$ close on $\left[x_{2 j}, x_{2 j+2}\right]$. Therefore, the integral over these intervals can also be made small since there are at most $L$ of them.

The next result shows that $\Psi$ preserves the homotopy class of a sweepout.

Lemma B.4 Let $\gamma \in \Omega$ satisfy $\max _{t} \operatorname{Energy}(\gamma(\cdot, t)) \leq L$. If $\gamma_{e}$ and $\tilde{\gamma}_{e}$ are given by applying Steps $\left(A_{1}\right)$ and $\left(A_{2}\right)$ to each $\gamma(\cdot, t),{ }^{12}$ then $\gamma, \gamma_{e}$ and $\tilde{\gamma}_{e}$ are all homotopic in $\Omega$.

Proof Given $x, y \in M$ with $\operatorname{dist}_{M}(x, y) \leq 4 \pi$, let $H(x, y):[0,1] \rightarrow M$ be the linear map from $x$ to $y$ as in (C2). It follows that

$$
F(x, t, s)=H\left(\gamma(x, t), \gamma_{e}(x, t)\right)(s)
$$

is an explicit homotopy with $F(\cdot, \cdot, 0)=\gamma$ and $F(\cdot, \cdot, 1)=\gamma_{e}$.

For each $t$ with Length $\left(\gamma_{e}(\cdot, t)\right)>0, \gamma_{e}$ is given by $\gamma_{e}(\cdot, t)=\tilde{\gamma}_{e}(\cdot, t) \circ P_{t}$ where $P_{t}$ is a monotone reparametrization of $\mathbf{S}^{1}$ that fixes $x_{0}=x_{2 L}$. Moreover, $P_{t}$ is continuous by (4.2) and $P_{t}$ depends continuously on $t$ by Lemma B.1. Since $x \rightarrow(1-s) P_{t}(x)+s x$ gives a homotopy from $P_{t}$ to the identity map on $\mathbf{S}^{1}$, we conclude that

$$
G(x, t, s)=\tilde{\gamma}_{e}\left((1-s) P_{t}(x)+s x, t\right)
$$

is an explicit homotopy with $G(\cdot, \cdot, 0)=\gamma_{e}$ and $G(\cdot, \cdot, 1)=\tilde{\gamma}_{e}$. Note that $P_{t}$ is not defined when Length $\left(\gamma_{e}(\cdot, t)\right)=0$, but the homotopy $G$ is.

\footnotetext{
${ }^{11}$ The speed is continuous because of the lower bound for the $a_{j}^{i}$ 's.

${ }^{12}\left(\mathrm{~A}_{1}\right)$ and $\left(\mathrm{A}_{2}\right)$ are defined in the beginning of Section 4.
} 
Acknowledgements We would like to thank Chris Croke for his comments. The authors were partially supported by NSF Grants DMS 0606629 and DMS 0405695.

\section{References}

[1] F J Almgren, The theory of varifolds, Mimeographed notes, Princeton (1965)

[2] G D Birkhoff, Dynamical systems with two degrees of freedom, Trans. Amer. Math. Soc. 18 (1917) 199-300 MR1501070

[3] G D Birkhoff, Dynamical systems, American Math. Soc. Coll. Publ. IX, American Mathematical Society (1927) MR0209095

[4] K Borsuk, Sur la courbure totale des courbes fermées, Ann. Soc. Polon. Math. 20 (1948) 251-265 MR0025757

[5] T H Colding, C De Lellis, The min-max construction of minimal surfaces, from: "Surveys in differential geometry, Vol. VIII (Boston, MA, 2002)", Int. Press, Somerville, MA (2003) 75-107 MR2039986

[6] T H Colding, W P Minicozzi II, Minimal surfaces, Courant Lecture Notes in Math. 4, New York University Courant Inst.of Math. Sciences, New York (1999) MR1683966

[7] T H Colding, W P Minicozzi II, Estimates for the extinction time for the Ricci flow on certain 3-manifolds and a question of Perelman, J. Amer. Math. Soc. 18 (2005) 561-569 MR2138137

[8] T H Colding, W P Minicozzi II, Width and finite extinction time of Ricci flow, Geom. Topol. 12 (2008) 2537-2586

[9] C B Croke, Area and the length of the shortest closed geodesic, J. Differential Geom. 27 (1988) 1-21 MR918453

[10] W Fenchel, Über Krümmung und Windung geschlossener Raumkurven, Math. Ann. 101 (1929) 238-252 MR1512528

[11] G Huisken, Flow by mean curvature of convex surfaces into spheres, J. Differential Geom. 20 (1984) 237-266 MR772132

[12] J Jost, Two-dimensional geometric variational problems, Pure and Applied Math. (New York), Wiley-Interscience Publ. John Wiley \& Sons Ltd., Chichester (1991) MR1100926

[13] G Perelman, Finite extinction time for the solutions to the Ricci flow on certain threemanifolds arXiv:math.DG/0307245

[14] J T Pitts, Existence and regularity of minimal surfaces on Riemannian manifolds, Math. Notes 27, Princeton University Press (1981) MR626027

[15] F Schulze, Evolution of convex hypersurfaces by powers of the mean curvature, Math. Z. 251 (2005) 721-733 MR2190140 
[16] L Simon, Lectures on geometric measure theory, Proc. of the Centre for Math. Analysis 3, Australian Nat. Univ. Centre for Math. Analysis, Canberra (1983) MR756417

[17] L Simon, Existence of surfaces minimizing the Willmore functional, Comm. Anal. Geom. 1 (1993) 281-326 MR1243525

Department of Mathematics, MIT

77 Massachusetts Avenue, Cambridge, MA 02139-4307, USA

and

Courant Institute of Mathematical Sciences

251 Mercer Street, New York, NY 10012, USA

Department of Mathematics, Johns Hopkins University

3400 N Charles St, Baltimore, MD 21218, USA

colding@math.mit.edu, minicozz@math.jhu.edu

Proposed: Ben Chow

Seconded: Colin Rourke, Martin Bridson
Received: 20 June 2007 Accepted: 10 October 2008 